Das Lignin ist demnach ein je nach den Voraussetzungen der allgemeinen und selektiven Kolloidadsorption und Gelbildung wechselndes Gemenge aus dem emährenden Kambialsaftstrom ausgeschiedener Kolloide, von welchen ein Teil reversibel, ein anderer Teil irreversibel in das Zellusosegerüst eingelagert ist.

Die im Holzkörper selbst vor dem Blattausbruch verhältnismäßig mächtig strömenden Blutungs- oder Frühjahrssäfte erscheinen dagegen nach ihrem Kolloidgehalt als ein Ergebnis der teilweisen Umkehrung dieser Adsorptionssynthese.

Diese Sätze sind durch die quantitative Ermittelung des Verhältnisses von adsorbierbaren Kolloiden zu nicht adsorbierbaren Kristalloiden in den beiden Hauptsaftarten experimentell erhärtet. Das genannte Verhältnis weist bei den
Blutungssäften einen verhăltnismäBig niedrigen und nur unbeträchtlich steigenden Kolloidgehait bis zur Zeit der Blattentfaltung auf. Der kambiale Bildungssaft ist dagegen zur Zeit der vegetativen Holzbildungstätigkeit außerordentlich reich an adsorbierbaren Kolloiden und diese nehmen gegen den SchluB der Jahresholzbildung im August stark ab. Die Safte reagieren mit chemisch indifferenten Oberflächenkörpern, wie Fasertonerde und Zellulose im Sinne der Adsorptionsisotherme.

$\mathrm{Ob}$ weitere Aufklärungen über die Natur des Lignins durch Anwendung einer fraktionierten quantitativen Adsorptionsanalyse erzielt werden können, werden weitere Versuche mit Pflanzensäften und mit besonders praparierter Sulfitlauge der Zelluloseindustrle ergeben.

\title{
Ueber Leitungshemmung durch Kolloide nebst Bemerkungen zum Serumleitvermögen.
}

Von Walter Frei, Rietheim (Schweiz). (Engtgangen 13. Dezember 1909)

\section{Einleitung.}

Wenn wir uns über die physikalischchemische Beschaffenheit physiologischer und pathologisch veränderter Körperflüssigkeiten Aufklärung verschaffen wollen, ist es notwendig, mit verschiedenen Methoden gleichzeitig vorzugehen. Denn jene Flüssigkeiten sind derart aufgebaut, das die meisten Methoden, einzeln angewandt, nicht ausschlieblich über die quantitativen und qualitativen Verhältnisse einer Komponente, auf die sie abgestimmt sind, AufschluB geben - wie z. B. die Leitfăhigkeit über Elektrolyte ...- sondern das Endresultat wird in höherem oder geringerem Grade auch von andern Bestandteilen des Versuchsobjektes beeinflubt. So sind die Ergebnisse einer der exaktesten Methoden, der Leitfähigkeitsmessungen, fast ebensosehr von den Kolloiden, wie von den Elektrolyten abhängig. Ueber die ersteren gibt die Viskositătsmessung Auskunft, deren Resultate hingegen auch in einem gewissen Grade durch lonen mitbedingt sind. Die osmotische Konzentration der Zwischenteilchenflüssigkeit eines Kolloidsystems (Serum) wird ziemlich genau - wenn wir vom osmotischen Druck der Kolloide ${ }^{1}$ ) absehen - durch die Gefrierpunkts-

1) Der osmotische Druck der Serumkolloide ist aller Wahrscheinlichkeit nach geringer als $40-50 \mathrm{~mm}$ depression angegeben, während das spezifische Gewicht von allen vorhandenen festen Bestandteilen summarisch-quantitativ Kenntnis gibt.

DasLeitvermögen einer homogenen wăssrigen Elektrolytlösung ist das Produkt des Dissoziationsgrades und der Summe der lonenbeweglichkeiten. Der erste dieser beiden Faktoren seinerseits ist abhängig von der Natur und Konzentration des Elektrolyten und von der Temperatur, ferner von Zusätzen von Elektrolyten mit gleichnamigen lonen (wobei Dissoziationsverminderung eintritt), wahrend Zugabe von Nichtelektrolyten (Zucker, Harnstoff) den Dissoziationsgrad im negativen Sinne beeinfluBt ${ }^{2}$ ). Der zweite Faktor ist, auBer durch Temperatur und Natur des Elektrolyten, bedingt durch die Reibung der Ionen am Lösungsmittel und am gelosten Stoff, außerdem durch anwesende Nichtelektrolyte, und zwar wird er -

$\mathrm{Hg}$, betraxchtliche Druckwerte, welche hingegen durch die Messung der Gefrierpunktserniedrigung mit dem Beckmannthermometer nicht mehr wahrgenommen werden konnen. Vgl. R. S. Lilli e, American Journal of Physiology 20, 127 (1907).

2) Kleine Zusatze von Nichtleitem zu stark dissozilerten Elektrolyten - wie es die Serumelektrolyte zum weitaus grobten Teil sind ... becinfluBten den Dissoziationsgrad unmerklich, hingegen bedeutend bei wenig dissoziierten Elektrolyten. Arrhenias, Ziitschr. f. physik. Chem. 9, 487 (1892). 
hauptsächlich wegen Erhöhung der lonenreibung durch dieselben - verkleinert ${ }^{3}$ ). Es existiert also eine Beziehung zwischen Leitfăhigkeit und innerer Reibung in dem Sinne, dab die eine zunimmt, wenn die andere vermindert wird ${ }^{4}$ ). Die Beziehungen der Leitfahigkeit zum osmotischen Druck und damit zur Gefrierpunktsdepression werden in sehr verdünnten Elektrolytgemischen, wie wir sie im (Salz-) Serum vor uns haben (wenn wir vom osmotischen Druck der Kolloide absehen), Rückschlüsse erlauben auf pathologische Konzentrationsveränderungen von Elektrolyten und Nonelektrolyten und Variationen des Verhältnisses der Konzentrationen dieser beiden Gruppen ${ }^{5}$ ). Gleichzeitiges Ansteigen oder Absinken der $\Lambda$ - und $\Delta$-Werte kündigt $\mathrm{Zu}$ nahme resp. Abnahme der lonenkonzentration an, wobei z. B. im letzteren Palle (mit Bezug auf Serum) Schwund von lonen vollstăndig aus der Blutflüssigkeit hinaus, oder aber Adsorption von seiten der Kolloidphase vorausgegangen sein kann. Verminderung des Leitvermögens unter gleichzeitigem starken Tiefersinken des Gefrierpunktes (beobachtet bei Piroplasmosis der Pferde \%), einer mit Blutkörperchenzerstörung einhergehenden Krankheit) kann nur durch Annahme einer beträchtlichen Erhöhung der Konzentration von nichtleitenden, osmotisch wirksamen Substanzen erklărt werden.

Das spezifische Gewicht reiner verdünnter Elektrolytlosungen (von ähnlieher Konzentration und Beschaffenhelt wie Salz-Serum) verändert sich - wenn wir die durch Elektrostriktion bedingten Veränderungen auBer acht lassen gleichsinnig mit der Leitfăhigkeit, bei Auftreten einer bedeutenden Quantităt von Nichtelektrolyten hingegen im Gegensinne.

Ist die Interionenflüssigkeit aber eine Kollo idlosung, so ist das Leitvermögen des ganzen Systems geringer als es ceteris paribus, d. h. nur von den oben erwähnten Paktoren abhăngig, sein würde ${ }^{7}$ ). Auch sind die Beziehungen der Leitfähigkeit zur Gefrierpunktsdepression, zur Viskosităt und zum spezifischen

9) Arrhenius, 1.c.

4) Vgl. Pissarjewsky und Karp, Zeitschr. f. physik. Chem. 68, 257 (1908), und Walden, ibidem 55, 207 (1906).

b) und 9 Vgl. W. Frei, Vergieichende physikal. chem. Blut- und Serumuntersuchungen usw. Zeltsch: fur Infektionskrankheiten usw. der Haustiere 6, 363 und $446(1909)$, sowle meine physikal.-chem. Untersuchungen tiber Piroplasmosis der Pferde, Ibidem, im Druck (1909).

7Vgl. Bugatszklund Tangl's und Oker-Blom's Untersuchungen, ref. in Hamburget, osmot. Druck usw.; Sch norf, Vet. med. Diss. (Zlirich 1904); Dumanski, Koll.-Zeitschr. 1, 300(1907) u. 2, Suppl.-Heft XVIII (1907).
Gewicht modifiziert. Hiervon soll im dritten Abschnitt die Rede sein.

\section{Experimentelles $\left.{ }^{8}\right)$.}

Die folgenden Experimente wurden hauptsächlich ausgeführt, um eine Idee zu gewinnen über den quantitativen EinfluB einiger organischer Kolloide verschiedener Konzentration, speziell der Serumalbumine und -globuline, sowie vergleichsweise auch von Gelatine und Saponin auf die Leitfähigkeit einer Lösung der lonen $\mathrm{Na}$ und $\mathrm{OH}$.

\section{Albumine.}

Ein Liter Pferdeserum wird mit 10 Liter destilliertem Wasser versetzt und die nach Absetzen der Globuline abgesogene klare Flüssigkeit nach Zusatz von etwas Ammonsulfat durch Kochen koaguliert. Das Koagulum wird unter Zuhilfenahme der Zentrifuge mit destilliertem $\mathrm{H}_{2} \mathrm{O}$ so lange gewaschen, bis die Leitfahigkeit des Waschwassers bei $37^{\circ} 0,75 \cdot 10^{-4}$ betrăgt und konstant bleibt. Das getrocknete Sediment dient zur Herstellung einer vierprozentigen Stammlobsung mit $\mathrm{n} / 2 \mathrm{NaOH}$, aus der durch Verdünnen mit $\mathrm{n} / 2 \mathrm{NaOH}$ die verschiedenen Konzentrationsabstufungen erhalten werden.

\begin{tabular}{|c|c|c|c|c|}
\hline \multicolumn{2}{|c|}{9.12 .07} & \multicolumn{3}{|c|}{$T=37^{\circ}$} \\
\hline $\begin{array}{c}\text { Konzentration } \\
\text { aee Albusmius } \\
\text { Proz. }\end{array}$ & $\Lambda \cdot 10^{-4}$ & $\begin{array}{l}\text { Absolute } \\
\text { Differens }\end{array}$ & $\begin{array}{c}\text { Differenz } \\
\text { Prox. }\end{array}$ & $\begin{array}{c}\text { Differenz } \\
\text { pro 1 Proz. } \\
\text { Kontentrat.- } \\
\text { Untersedhied } \\
\text { d. Kolloiddes } \\
\text { Proz. } \\
\end{array}$ \\
\hline $\begin{array}{c}0(\mathrm{n} / 2 \mathrm{NaOH}) \\
0,5 \\
1,0 \\
1,5 \\
2,0 \\
2,5 \\
3,0 \\
3,5 \\
4,0\end{array}$ & $\begin{array}{r}1097 \\
1056 \\
1015 \\
977 \\
942 \\
905 \\
870 \\
837 \\
802\end{array}$ & $\begin{array}{l}41 \\
41 \\
38 \\
35 \\
37 \\
35 \\
33 \\
35\end{array}$ & $\left.\begin{array}{l}\left.\begin{array}{l}3,7 \\
3,9\end{array}\right\} \\
3,7 \\
3,7\end{array}\right\}$ & $\begin{array}{l}7,6 \\
7,4\end{array}$ \\
\hline
\end{tabular}

Die Verminderung von $\Lambda$ bei anwachsender Albuminkonzentration nimmt absolut ab, prozentual zu und beträgt im Mittel für 1 Proz. Zunahme der Kolloidkonzentration ca. 7,7 Proz.

Der Rest der Stammlősung : wird durch Kochen sterilisiert und über Nacht im Eiskasten r.afbewahrt.

i0. 12. 07. $\Lambda$ der Stammlösung $=786 \cdot 10^{-4}$ d. h. die Leitfähigkeit hat um ca. 2 Proz. abgenommen, wahrscheinlich infolge Ionen-

8) Die Experimente wurden s. Z. im bakteriolog. Institut zu Pretoria ausgefuhrt. 
adsorption. Das Experiment vom 9.12, wird wiederholt. Die Leitfähigkeit der Stammlösung bleibt während der Dauer des Versuches konstant:

\begin{tabular}{|c|c|c|c|c|}
\hline Stammlösung & & $\begin{array}{l}\text { Zeit } \\
.30 \text { a m } \\
, 45 \text { a m } \\
00 \mathrm{pm} \\
, 15 \mathrm{pm}\end{array}$ & A. 10 & $\begin{array}{l}0^{-4}\left(37^{0}\right) \\
785 \\
787 \\
785 \\
786\end{array}$ \\
\hline $\begin{array}{c}\text { Konzentration } \\
\text { des Albumins } \\
\text { Proz. }\end{array}$ & $\wedge .10^{-4}$ & $\left|\begin{array}{c}\text { Absolute } \\
\text { Differenz }\end{array}\right|$ & $\begin{array}{c}\text { Differenz } \\
\text { Proz. } \\
\end{array}$ & $\begin{array}{l}\text { Differenz } \\
\text { pro 1 Proz. } \\
\text { Kanzentrat.- } \\
\text { Unterschled } \\
\text { d. Kolloides } \\
\text { Proz. } \\
\end{array}$ \\
\hline $\begin{array}{c}0(\mathrm{n} / \mathrm{s} \mathrm{NaOH}) \\
0,5 \\
1,0 \\
1,5 \\
2,0 \\
2,5 \\
3,0 \\
3,5 \\
4,0\end{array}$ & $\begin{array}{r}1095 \\
1055 \\
1015 \\
974 \\
935 \\
898 \\
860 \\
823 \\
786\end{array}$ & $\begin{array}{l}40 \\
40 \\
41 \\
39 \\
37 \\
38 \\
37 \\
37\end{array}$ & $\left.\begin{array}{l}3,7 \\
3,8 \\
4,0 \\
4,0 \\
4,0 \\
4,2\end{array}\right\}$ & $\begin{array}{l}7,5 \\
8,0\end{array}$ \\
\hline
\end{tabular}

Dasselbe Kolloid in denselbet Konzentrationen hemmt heute stärker als gestern, d. h. die Spuren seiner Erlebnisse (Sterilisieren, Aufenthalt im Eiskasten) sind auch durch die Aenderung der Leitfăhigkeit erkennbar (Hysteresis).
2. Globuline.

Die durch Verdinnen des Serums (siehe oben) gefallten Globuline werden so lange gewaschen, bis das Leitvermögen des Waschwassers auf den konstant bleibenden Went von $\Lambda=0,2 \cdot 10^{-4}$ angelangt ist. Aus den getrockneten Globulinen wird mit $\mathrm{n} / 2 \mathrm{NaOH}$ eine dreiprozentige Stammlösung hergestellt, welche nach 48 stündigem Aufenthalt im Eisschrank zur Untersuchung gelangt.

\begin{tabular}{|c|c|c|c|c|}
\hline \multicolumn{3}{|c|}{ 13. 12.07} & \multicolumn{2}{|l|}{$T=37^{\circ}$} \\
\hline & $9,30 \mathrm{am}$ & $10,00 \mathrm{am}$ & $12,30 \mathrm{pm}$ & $1,00 \mathrm{pm}$ \\
\hline 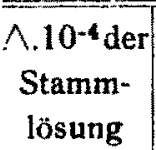 & 863 & & 888 & \\
\hline $\begin{array}{c}\text { A.10 } 0^{-4} \text { der } \\
\mathrm{NaOH}- \\
\text { losung }\end{array}$ & & 1094 & & 1093 \\
\hline
\end{tabular}

Die Leitfähigkeit der Stammlösung nimmt also während der Versuchsdauer, $d$. h. dreistündigem Aufenthalt bei Zimmertemperatur (ca. $30^{\circ}$ ), um etwa 3 Proz, zu (Hysteresis, vielleicht durchDissoziation derlonenkolloidverbindungen), wodurch der Hemmungseffekt des Kolloids um einen kleinen Betrag herabgemindert wird.

13. 12. $07 \quad \mathrm{~T}=37^{\circ}$

\begin{tabular}{|c|c|c|c|c|}
\hline $\begin{array}{c}\text { Konzentration } \\
\text { des Globulins } \\
\text { Proz. }\end{array}$ & $\wedge .10^{-4}$ & Absolute Differenzen & $\begin{array}{c}\text { Differenzen } \\
\text { Proz. } \\
\end{array}$ & $\begin{array}{c}\text { Differenz pro } 1 \text { Proz. } \\
\text { Konzentrationsunterschied } \\
\text { des Kolloids } \\
\text { Proz. }\end{array}$ \\
\hline $\begin{array}{c}0(\mathrm{n} / 2 \mathrm{Na} \mathrm{OH}) \\
0,5 \\
1,0 \\
1,5 \\
2,0 \\
2,5 \\
3,0\end{array}$ & $\begin{array}{r}1094 \\
1057 \\
1023 \\
988 \\
954 \\
921 \\
888\end{array}$ & $\begin{array}{l}37 \\
34 \\
35 \\
34 \\
33 \\
33\end{array}$ & $\left.\begin{array}{l}3,4 \\
3,2 \\
3,4 \\
3,4 \\
3,5 \\
3,6\end{array}\right\}$ & $\begin{array}{r}6,6 \\
6,8 \\
7,1\end{array}$ \\
\hline
\end{tabular}

Die Serumglobuline setzen die Leitfähigkeit weniger herab als die Albumine.

\section{Gelatine.}

Am 3. 1. 07 wird mit $\mathrm{n} / 2 \mathrm{NaOH}$ eine zweiprozentige Stammlösung hergestellt, welche bis zum $7.1 .08 \mathrm{im}$ Eiskasten verweilt.

$\begin{array}{ccc}\text { 7. 1. } 08 \quad \mathrm{~T}=37^{\circ} & \\ & \text { Zeit } & \wedge .10^{-4} \\ \text { Zweiprozentige } & 11,30 \mathrm{am} & 980 \\ \text { Gelatinestammlösung } & 12,00 & 980 \\ & 6,00 \mathrm{pm} & 973 \\ & 6,20 \mathrm{p} \mathrm{m} & 972 \\ \mathrm{n} / 2 \mathrm{NaOH} \text {-Lósung } & 10,30 \mathrm{am} & 1099 \\ & 6,40 \mathrm{pm} & 1100\end{array}$


8. 1. 08. $\mathrm{T}=37^{\circ}$

\section{Zweiprozentige}

Gelatinestammlösung, Zeit $\wedge .10^{-4}$ über Nach: im Eiskasten

aufbewahrt worden $10,30 \mathrm{am} \cdot 954$
Durch siebenstündigen Aufenthalt bel Zimmertemperatur (ca. $30^{\circ}$ ), d. h. während der Versuchsdauer, nimmt $\wedge$ um ca. 0,8 Proz. und bei darauffolgendem 16 stündigen Verweilen im Eisschrank um weitere 1,85 Proz. ab (Hysterese, vgl. Albumine und Globuline).

7. 1.08

$r=370$

\begin{tabular}{|c|c|c|c|c|c|}
\hline $\begin{array}{c}\begin{array}{c}\text { Konzentration } \\
\text { der Gelatine } \\
\text { Proz. }\end{array} \\
\end{array}$ & $\wedge .10^{-4}$ & $\begin{array}{c}\text { Absolute } \\
\text { Differenzen }\end{array}$ & $\begin{array}{c}\text { Differenzen } \\
\text { Proz. }\end{array}$ & $\begin{array}{c}\text { Differenz pro } 1 \text { Proz. } \\
\text { Unterschied der } \\
\text { Kolloidkonzentration } \\
\text { Yroz. }\end{array}$ & $\begin{array}{l}\text { Viskositat } \\
\text { bei } 30^{\circ}\end{array}$ \\
\hline $\begin{array}{c}0 \mathrm{n} / 2 \mathrm{NaOH}) \\
0,25 \\
0,5 \\
0,75 \\
1,00 \\
1,25 \\
1,50 \\
1,75 \\
2,00\end{array}$ & $\begin{array}{r}1099 \\
1082 \\
1068 \\
1054 \\
1039 \\
1025 \\
1008 \\
989 \\
973\end{array}$ & $\begin{array}{l}17 \\
14 \\
14 \\
15 \\
14 \\
17 \\
19 \\
16\end{array}$ & $\left.\begin{array}{l}1,5 \\
1,3 \\
1,3 \\
1,4 \\
1,3 \\
1,7 \\
1,9 \\
1,6\end{array}\right\}$ & 5,5 & $\begin{array}{l}1,08 \\
1,10 \\
1,10 \\
1,11 \\
1,12 \\
1,16 \\
1,16 \\
1,18 \\
1,20\end{array}$ \\
\hline
\end{tabular}

4. Saponin.

Gleichzeitig mit der Gelatinestammlősung (3.1.08) wird eine vierproz. Saponinlösung in $\mathrm{n} / \mathbf{2} \mathrm{NaOH}$ bereitet und unter genau denselben auBeren Bedingungen gehalten wie jene. Im Gegensatz zu derselben, sowie zu den Albuminund Globulinlösungen zeigt die Saponinstamm18sung äuberen, speziell thermischen Binflüssen gegenüber eine grofe Unempfindlichkeit, die sich in Konstantbleiben der Leitfähigkeit kundgibt:

$$
\begin{aligned}
& \mathrm{T}=37^{\circ} \\
& \text { Zeit } \wedge .10^{-4}
\end{aligned}
$$

\begin{tabular}{|c|c|c|c|c|c|}
\hline $\begin{array}{c}\begin{array}{c}\text { Saponin- } \\
\text { Konzentration } \\
\text { Proz. }\end{array} \\
\end{array}$ & $\wedge \cdot 10^{-4}$ & $\begin{array}{c}\text { Absolute } \\
\text { Differenzen }\end{array}$ & $\begin{array}{c}\text { Differenzen } \\
\text { Proz. } \\
\end{array}$ & $\begin{array}{c}\text { Differenz pro } 1 \text { Proz. } \\
\text { Differenz der } \\
\text { Kolloidkonzentration } \\
\text { Proz. }\end{array}$ & $\begin{array}{l}\text { Visknsitat } \\
\text { bei } 30^{\circ}\end{array}$ \\
\hline $\begin{array}{c}0(\mathrm{n} / \mathbf{2} \mathrm{NaOH}) \\
0,5 \\
1,0 \\
1,5 \\
2,0 \\
2,5 \\
3,0 \\
3,5 \\
4,0\end{array}$ & $\begin{array}{r}1096 \\
1065 \\
1035 \\
1007 \\
979 \\
953 \\
928 \\
903 \\
881\end{array}$ & $\begin{array}{l}31 \\
30 \\
28 \\
28 \\
26 \\
25 \\
25 \\
22\end{array}$ & $\begin{array}{l}2,8 \\
2,8 \\
2,7 \\
2,8 \\
2,7 \\
2,6 \\
2,7 \\
2,4\end{array}$ & $\begin{array}{r}5,6 \\
5,5 \\
5,3 \\
5,1\end{array}$ & $\begin{array}{l}1,08 \\
1,10 \\
1,12 \\
1,18 \\
1,18 \\
1,18 \\
1,20 \\
1,22 \\
1,23\end{array}$ \\
\hline
\end{tabular}

$$
\text { 7. 1. } 08 \quad \mathrm{~T}=37^{\circ}
$$

Im Gegensatz zu den drei vorhergehenden Kolloidarten nimmt die Verminderung der Leitfahigkeit bei steigender Saponinkonzentration nicht nur absolut, sondern auch prozentual ab.

\section{Theoretisches.}

In den nun folgenden Betrachtungen möchte ich an Hand der mitgeteilten Experimente mit einfachen heterogenen hydrophilen Systemen zeigen,

1. von welchen Faktoren das Endresultat einer Messung des Serumleitvermbgens abhängt und alsdann

2. welche Rückschlüsse sich aus den Ergebnissen solcher Messungen ziehen lass en auf physikalisch chemsche Zusammens tung 
und pathologischeVeränderungen von Seren,

anderer Methoden (Viskosimetrie, Kryospeziell bei gleichzeitiger Zuhilfenahme skopie, spezifische Gewichtsbestimmungen). Leitfahigkeit und Leitfähigkeitsabnahme in verschiedenen Kolloiden
varrijerender Konzentration.

Leitfähigkeit der $\mathrm{n} / \mathrm{z} \mathrm{NaOH}$-Lösung $=1097 \cdot 10^{-4}$

\begin{tabular}{|c|c|c|c|c|c|c|c|c|}
\hline \multirow[b]{2}{*}{ Konzentration } & \multicolumn{4}{|c|}{ Leitfähigkeit $10^{-4}$} & \multicolumn{4}{|c|}{$\begin{array}{l}\text { Abnahme der Leitfahigkeit in Prozenten } \\
\text { pro Prozent der Kolloidkonzentration }\end{array}$} \\
\hline & $1 \%$ & $2 \%$ & $3 \%$ & $4 \%$ & 1. & 2. & 3. & 4. \\
\hline $\begin{array}{l}\text { Serumalbumin } \\
\text { Serumglobulin } \\
\text { Gelatine } \\
\text { Saponin }\end{array}$ & $\begin{array}{l}1015 \\
1023 \\
1089 \\
1035\end{array}$ & $\begin{array}{l}935 \\
954 \\
973 \\
979\end{array}$ & $\begin{array}{l}860 \\
888 \\
928\end{array}$ & 881 & $\begin{array}{l}7,5 \\
6,6 \\
5,5 \\
5,6\end{array}$ & $\begin{array}{l}8,0 \\
6,8 \\
6,5 \\
5,5\end{array}$ & $\begin{array}{l}8,2 \\
7,1 \\
5,3\end{array}$ & 8,8 \\
\hline
\end{tabular}



Aus den Tabellen ist zu ersehen, das alle untersuchten Kolloide das Leitvermögen der $n / 2 \mathrm{NaOH}$-Losung in beträchtlichen, jedoch auch bei gleichen Konzentrationen - ungleichem MaBe herabsetzen ${ }^{9}$ ). Es übt also jedes Kolloid eine spezifische Hemmung aus. Der absolute Betrag der Leitfăhigkeitsabnahme nimmt mit steigender Kolloidkonzentration -. Gelatine ausgenommen - ab, während in Prozenten des jeweilig yorausgehenden Leitfähigkeitswertes ausgedrückt die Gröbe dieser Abnahme bei je 1 Proz.

9) Siehe letzte Tabelle und Kurve.
Konzentrationszunahme des Kolloids anwächst (hier bildet Saponin eine Ausnahme).

Wir wollen uns nun uber die bei der Ausführung der oben mitgeteilten Experimente im Innern des Kolloidsystems stattfindenden physikalisch-chemischen Vorgänge prinzipiell Klarheit zu verschaffen suchen, um dadurch zum Verstehen des endlichen Resultates zu gelangen.

In der reinen wässerigen $\mathrm{n} / \mathbf{2} \mathrm{NaOH}$-Lösung sind die Ionen $\mathrm{Na}$ und $\mathrm{OH}$ und die Moleküle $\mathrm{NaOH}$. Durch Einführung der dispersen Phase in das System wird das Dissoziationsgleichgewicht durch sofort einsetzende Quellungs- 
und Adsorptionsurozesse gestört; denn eitumal wird durch Quellungswasseraufnahme von seiten der Kolloidtellchen die Menge des Solvens reduziert ${ }^{10}$ ) und dadurch die Konzentration des Gelosten erhöht, andererseits verschwindet eine gewisse Anzahl von lonen durch Adsorption aus der Zwischenflüssigkeit ") wodurch diese verarmt und der Dissoziationskoeffizient vetandert wird ${ }^{12}$ ). Die Losung wird wohl nicht tel quel von den Teilchen aufgenommen, sondern es werden, wie dies durch $\mathrm{H}$ o $\mathrm{m}$ e is t e r experimentell an gequollenem Leim gezeigt (der verhalltnismäBig mehr Salz als Wasser aufnimmt, so $\mathrm{da} B$ die Konzentration der aufgesaugten Lobsung hơher ist als die der dargebotenen Flüssigkeit) und nach den theoretischen Ausfóhrungen Kaufler's (wonach die Konzentration auf der konkaven Seite einer permeablen Oberflache immer grober sein muB), zu erwarten ist, wohl auch von den von uns angewandten Kolloiden verhaltnismäbig mehr lonen aufgenommen werden. Der Eintritt von Ionen in die disperse Phase findet also nicht einfach nach MaBgabe der Lóslichkeit, d. h. nach dem Verteilungsgesetz, statt, sondern wird måchtig durch Oberflächenkräfte beeinflubt.

Dadurch daB ein Ion mehr als ein anderes adsorbiert wird, kommen an den Grenzflachen Potentialdifferenzen zustande (elektrische Doppelschichten), welche auf die Oberflachenspannungen und damit auf Adsorptionsprozesse an den Grenzflăchen einwirken. Diese Verhältnisse kommen namentlich bei Konzentrationsveranderungen in der Zwischenflüssigkeit, also auch in der Blutflüssigkeit, wo ja bestăndiger Wechsel der Bestandteile herrscht, zur Geltung.

10) Es geht also eine bestimmte Menge Wassers für Ionen und dadurch für den "Leiterquerschnitt" verloren. Diese Wassermenge wird zur Quellung, d.h. zur Vergroberung des Gelatinequerschnitles gebraucht. Aus dlesem Grunde kann derselbe nicht einfach aus dem absoluten $(=\mathrm{g})$ spezifischen Gewicht (c) der

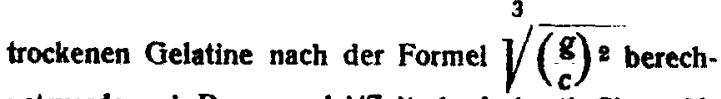
net werden,wie Dum a n sk i(Zeitschr. f. physik. Chem. 60, 553 (1907) und Koll.-Zeitschr. 2, I.Suppl.-H. XVIII (I907) meint, sondern er ist grober.

11) Aehnliche Prozesse spielen sich in der Blutflissigkeit ab, wenn durch pathologische Vorgänge netie Kolloide in den Blutstrom gelangen, $z$. B. bei Piroplasmosis der Pferde.

12) Die $\mathrm{n} / \mathrm{g} \mathrm{NaOH}$-Losung ist ungefahr zu 90 Proz. dissoziiert. Ich nehme an, das mehr lonen adsorbiert werden als nach der Storung des Dissozlationsgleichgewichtes durch erneute Dissoziation der noch nicht dissoziterten $\mathrm{NaOH}$-Molekule frel werden, wodurch also die Flussigkeit tatsächlich an lonen verarmt.
Es wird sich nach all diesen erwăhnten Quellungs- und Adsorptionsvorgangen in der Stammlosung eine Art Gleichgewicht zwischen den Konzentrationen in den beiden Phasen herstellen. Das Leitvermögen des zur Ruhe gekommenen Systems ist kleiner als dasjenige der reinen Elektrolytlossung, weil

1. durch Einführung der dispersen Phase eine Verkleinerung des Leiterquerschnittes (nach Du m a n s ki) stattgefunden hat,

2. die Anzahl der Elektrizităt transportierenden Elemente, der lonen, in der Zwischenflüssigkeit reduziert ist ${ }^{18}$ ),

3. die Kolloidteilchen als mechanische Hindernisse der Wanderung der lonen im Wege stehen und außerdem

4. durch Erhöhung der innern Reibung deren Beweglichkeit vermindern.

Diese Faktoren sind auch im Serum vorherrschend und bewirken, dab seine Leitfahigkeit bedeutend geringer ist als sie nach Menge und Art der darin enthaltenen Elektrolyte elgentlich sein sollte.

Bei der Herstellung der einz'Inen Proben verschiedener Kolloid-Konzentration wird beim Experimentieren die Stammlosung mit einer entsprechenden Menge von $\mathrm{n} / 2 \mathrm{NaOH}$-Lösung verdünnt, d. h. die Flüssigkeit zwischen den Teilchen wird bei jeder niedrigeren Konzentrationsstufe des Kolloids reicher an $\mathrm{Na}$ und $\mathrm{OH}$, weshalb jedesmal von neuem lonen von den einzelnen Teilchen adsorbiert werden, hingegen jedesmal etwas weniger. Denn denken wir uns z. B. zu einer bestimmten Menge Stammlösung eine kleine Quantităt von $\mathrm{n} / 2 \mathrm{NaOH}$ Lösung zugesetzt, so sind relativ viele Teilchen, welche Ionen adsorbieren, die Zwischenflussigkeit wird also relativ stark verarmen. Gibt man hingegen sehr viel der $n / 2 \quad \mathrm{NaOH}-$ Lösung zu, so sind verhältnismăBig wenig adsorbierende Partikelchen vorhanden, weshalb die Zwischenfluissigkeit auch sehr wenig lonen verliert. Mit abnehmender Kolloidkonzentration steigtalso die lonenkonzentration in der Zwischenflüssigkeit, d. h. es superponieren sich zwei die Leitfahigkeit stark begünstigende Momente. Zudem kommt noch ein weiterer forderlicher Umstand, nămlich die Abnahme der inneren Reibung des Systems infolge Verringerung der Konzentration des Kolloids. Endlich ist noch zu erwähnen, daß die obengenannte, bei jeder

18) Die adsorbierten Ionen sind nur mehr indirektan der Stromleitung dadurch beteiligt, daB sie die Elgenleitfahigkeit des Kolloids durch Vergroberung der Teilchenladung erhoben. (Siehe unten.) 
7ugahe von $\mathrm{n} / 2 \mathrm{NaOH}$-Lobsung von neuem stattfindende Adsorption von Ionen an die Teilchen deren ladung und damit die Eigenleitfähigkeit des Kolloids vergröbert. Hingegen involviert die erhohts Korzentration im Teilchen eine erncute Wasseraufnahme ${ }^{14}$ ) und die dad irch hervorgerufene Volumvergrötlerung beeinfluAt die Leitfahigkeit im negativen Sinne. Rekapitulierend können wir die bis jetzt betrachteter Faktoren, the bei Verdünnungen des Kolloids mit der imm:r gleich konzentrierten Elektrolytlösung folgendemafen zusammenstellen :

Einen günstigen Eüfluh auf die Leitfähigkeit haben

1. Konzentrationsabuahme des Kolloids.

2. Erhöhung der lonenkonzentration in der Zwischenflüssigkeit,

3. Erhöhung der Eigenleitfähigkeit derTeilchen,

4. Verminderung der inneren Reibung.

Gleichzeitig üben aber einen ungünstigen Einflu $\mathrm{B}$ auf die Leitfahigkeit aus:

1. Anwachsen des Teilchenvolums,

2. fernere Adsorption von Ionen.

Die ersien vier Faktoren überwiegen, die Leilfăhigkeit wächst schncller an als nur der Abnahme der Kolloidkonzentration entsprechend, d. h. die Kurven sind leicht konkav.

Der Vollständigkeit halber muB hier noch eines 'Faktors gedacht werden, der - wenn auch in geringem Grade nur - die Resultate unserer Leitfahigkeitsmessungen mit beeinfluBt, der Kataphorese und im Zusammenhang damit der Elektróendosmose und der Strömungsströme. In einem Kolloide entstehen durch Bewegung der Teilchen Strömungsströme (Quincke). Bilden die Teilchen ein unbewegliches Gerüstwerk, dessen Maschen von der Flüssigkeit durchtränkt sind, so bewegt sich diese, wenn man einen Strom durch das System leitet (Elektroendosmose). Sind die Partikelchen aber lose, so werden sie durch den Strom zu einer der Elektroden transportiert (Kataphorese), wobei aber nicht gesagt ist, daB die Flüssigkeit still steht (was den andern Grenzfall darstellen wârde). Vielmehr ist man berechight anzunehmen, dab auch die Flüssigkeit in einen zit Bewegungsrichtung der Teilchen entyegengesetzten Sinne verschoben wird. It ansern Experimenten

14) Wahrscheinlich werder dit Quellungsbedingungen, welche anfangs durch Alkali aüchtig beginnstigt werden, mit zunehmender OH-Konzentration immer ungünstiget, da unsere Konzentrationen bereits jenselts der optimalen liegen dürten. Vgl. Wo. Ostwald Pfliuger's Arch. 108, 563 (1905).

werden also im System folgende Bewegungen stattfinden:

$$
\begin{aligned}
& \text { Zur Anode: } \\
& \text { OH }
\end{aligned}
$$

Kolloidteilchen

Es leuchtet also ohne weiteres ein, dak die innere Reibung des Lösungsmittels ${ }^{15}$ ) und die Reibung der Teilchen an demselben, $\mathbf{d} . \mathbf{h}$. die Viskosität des ganzen Systems, bei diesen Vorgängen bedeutende Rollen spielen. Der Durchmesser der Teilchen (Quellungsgrad), besser gesagt die Gröbe ihrer Oberfläche, wird auf die Geschwindigkeit ihrer Fortbewegung einen Einfluß ausüben, welcher sich nach Matgabe der Einwirkung von Konzentrationsverschiebungen der Ionen in der Zwischenflüssigkeit verändert, da der Quellungsgrad jedenfalls bei verschiedener Konzentration auch derselben Elektrolyten ein anderer ist. Endlich (mit Rücksicht auf Serum) beeinflussen schon geringe Ionenzusătze die Transportschnelligkeit hydrophiler Kolloide ganz bedeutend (Perrin ${ }^{16}$ ) durch Veranderung der Stärke der elektrischen Ladung.

Die Eigenleitfähigkeit eines Kolloides hăngt also von einer Reihe von Faktoren ab: Reibung der Teilchen an der Plüssigkeit, Quellungsgrad der crsteren und damit von der Ausdehnung und der Beschaffenheit der Oberfläche, vom spezifischen Gewicht der Partikelchen, von ihrer Ladungsgröbe ${ }^{17}$ ) die ihrerseits durch die Art und Konzentration der lonen in der Zwischenflitssigkeit mitbedingt wird.

Praktisch kommt die Eigenleitfahigkeit des Kolloids, z. B. bei Serumuntersuchungen, kaum in Betracht, da sie, verglichen mit dem durch die lonen besorgten Elektrizitätstransport, sehr gering ist, andererseits durch die sehr enorine Hemmwirkung der Kolloide weit in den Hintergrund gedrängt wird.

Die vorausgegangenen Betrachtungen über die physikalisch-chemischen Vorgănge, die in einem Kolloidsystem sich abspielen und die Leitfähigkeit desselben bestimmen, finden auch

15) Vgl. die lonenhullen, die vom Losungsmittel gebildet werden, $H$. C. Jones und W. R. Veczey, Zeitschr. f. physik. Chem. 61, 641 (1908). (IonenSolvens - Assoziationen, Kohlrausch.)

16) Compt. rend. 136, 1388 (1903); 137, 513 und 564 (1903); zit.n. A. Muller, Allg. Chemie der Kolloide. Die Transportschnelligkeit wird durch Ionen gleicher Ladung gefördert, durch solche gegensinniger Ladung verringert, $B$ urt on, Phil. Mag. (4) 11, 425, 12, 472 (1906); zit. n. Lewis, Koll.-Zeitschr. 4, 209 (1909).

17) Die Grösse der Ladung ist um so grosser, je ausgedehnter die Gesamt-Oberfiache, d. h. Je kleiner die Teilchen. Ducla $1 \mathrm{x}$, Koll. - Zeitscht. 3, 126 (1908). 
im Serum statt, nur sind hier die Verhältnisse noch bedeutend komplizierter, weil wir ein Gemisch verschiedener Arten von Ionen und von Kolloiden vor uns haben. Die Leitfahigkeit des Serums kann natürlich nur ein unvollkommenes Bild von den Vorgängen in der "lebenden" Blutflüssigkeit geben; denn sie ist der Ausdruck eines ephemeren, eigentlich nur einen Moment dauernden Zustandes, der selbst wieder das Produkt einer ununterbrochenen Kette physikalisch-chemischer Prozesse, der Vorgeschichte ist. Das Leitvermögen des -ungefähr 8 Proz. Eiweif enthaltenden - Serums ist betrăchtlich, nämlich 20 Proz. geringer als das des Salzserums ${ }^{18}$ ). Es ist in erster Linie bedingt durch die Konzentration freier Ionen und den Gehalt an Eiweifkörpern, d. h, von zwei konkurrierenden Momenten, weshalb also bei Abnahme der ersteren oder Zunahme der letzteren die Leitfähigkeit abnimmt ${ }^{19}$ ). Finden beide Prozesse gleichzeitig in dem angegebenen Sinne statt, so superponieren sich ihre Wirkungen, walhrend, wenn lonen und Kolloide gleichzeitige und gleichsinnige Konzentrationsschwankungen erleiden, ihre Einflüsse auf die Leitfahigkeit einander paralysieren kơnnen. Solche Veränderungen können aber durch Heranziehen anderer Methoden erkannt werden (Viskosimetrie, Bestimmung des spez. Gew.).

AuBer der Menge ${ }^{20}$ ) der Serumkolloide haben natürlich auch ihr Quellungszustand, Größe und Beschaf:enheit der Teilchen, eventuelle Struktur oder Konglomerationsgrad EinfluB auf den Leitwiderstand, und diese Momente sind ihrerseits von den lonen des $Z$ wischenwassers abhängig. So erhohen z. B. die lonen $\mathrm{H}$ und $\mathrm{OH}$ die Aufnahme von Quellungswasser (Wo. Ostwald, Lillie. M.H.Fis c her), und Konzentrationsveränderunger, schon dieser beiden lonen (wie sie zu Krank-

18) Bugarszki u. Tangl (n. Hamburger) fanden eine Emiedrigung des Leltvermogens von 2,5 Proz. pro Gewichtsprozent Eiweik, also eine bedeutend getingere Herabsetzung als meine Serumalbumine und -globuline sie verursachen. Dieser Unterschied erklärt sich aus der Verschiedenheit der Art und Konzentration der angewandten Elektrolyte; diese Autoren experimentierten nämlich mit den Serumelektrolyten in natürlicher Konzentration.

19) Durch Zuhilfenahme der Viskosimetrie, die ja speziell iber Schwankungen des Kolloidgehaltes Auskunft gibt, kann erkannt werden, ob Aenderungen der Leitfähigkeit durch Variationen der Ionen oder Kolloidkonzentration hervorrgerufen sind. (Speziell bei der Deutung der Resultate an pathologischen Seren angezeigt.)

20y Menge als Trockensubstanz gedacht. Das Quellungswasser gehort natürlich als unerlä日licher Bestandteil zum Begriff Kolloid. heitszeiten häufig vorkommen) werden auf die erwähnte Weise indirekt den elektrischen Widerstand beeinflussen. Die Ionen der Neutralsalze werden in versthiedenem Grade adsorbiert und wirken auch ungloich (in spezifischer Weise, auf den Quellungszustand (und damit auf den Leitungswiderstand) ein. Die im Gefolge auch des normal ablaufenden Stoffwechsels andauernd stattfindenden Konzentrationsschwankungen von Ionen werden nicht nur die Leitfähigkeit des Zwischenteilchenwassers veräıdern, sondern durch beständiges Auf- und Abquellen der Kolloide indirekt dic I eitfähigkeit des ganzen Systems beeinflussen.

Pathologischerweise können im Serum neue, fremde Substanzen auftreten, Kolloide ( $z, B$. von zerstörten Blutkörperchen herrïhrend,Stromabestandteile oder Hâmoglobin), die das Leitvermögen ganz bedeutend herabsetzen ${ }^{21}$ ); auch Gallenbestandteile (gallensaure Salze, Biliverdin) werden nicht wirkungslo; sein. Eine bedeutende Ansammlung von Nichtelektrolyten (bei Piroplasmosis der Pferte) könnte durch starke Zurückdrängung der Dissoziation Elektrolytsciiwund vortäuschen. Gleichzeitige Bestimmung der Gefrierpunktsdepression bewahrt jedoch vor solchem Fehlschluh.

Zu zehn tatsächlich beobachteten ${ }^{22}$ ) gleich zeitig) stattgefundenen Veränderungen von Leitfahigkeit, Gefrierpunktsdepression und spezifischem Gewicht und zu fünfzehn Pällen synchroner Schwankungen von Leitfähigkeit, Viskositat und spezifischem Gewicht versuchte ich folgende Deutungen:

1. $\Lambda$ und $\triangle$ ändern sich nicht, s fällt. Das bedeutet Abnahme der Kolloid- und Ionen-Konzentration; Quantitätsverminderung der Kolloide allein würde VergröBerung von $\wedge$ und $\triangle$ zur Folge haben.

2. $\wedge$ und $\Delta$ bleiben konstant s steigt an: Die Ursache ist Anwachsen des Kolloid- und Elektrolytgehaltes.

2) Es genügt nicht, nur eine einzige Serumprobe bei einer Krankheit 7.4 untersuchen, da die absolute Wertgrobe nicht immer anzeiyt, of sie pathologisch oder normal sei. Werfe, die für ein Individuum pathologisch sind, kơnnen fir ein anderes noch innerlalb der Grenzen des Normalen iiegen. Deshalb haben wir, um den normalen individuellen Durchschntt $2 \mathrm{u}$ kennen, unsere Benbachtungen (bei Piroplasmosis und Pferdesterbe) immer einige Tage vor der (kunstlichen) Infektion begonnen.

*) Zahlen siche meine .Vergleichendèn physikal. chem. Blut- und Serumuntersuchungen usw." Zeitschr. f. Infektionskrankheiten usw. der Haustiere 6, 363 und 446 (1909); auBerdem W. Frei, Physikal.-chem. Untersitchungen iber Piroplasmosis det Pferde, ibidem. 
3. $\wedge$ bleibt unverändert, $\Delta$ und s nehmen zu: Bedeutendes Ansteigen der Konzentration der Nichtelektrolyte; um die dadurch bedingte Herabsetzung der Dissoziation zu paralysieren, ist eine Erhöhung des Ionengehaltes notwendig.

4. $\wedge$ nimmt beträchtlich $a b, s$ bedeutend $z u$, $\triangle$ andert sich nicht oder nur wenig: Der Kollokdgehalt des Serums, sowie die Konzentration der Nichelektrolyte haben zugenommen; die Konsequenz ist beträchtliche Adsorption und Hinderung der lonenwanderung, auBerdem $\mathrm{Zu}$ rückdrängen der Dissoziation durch die Nichtelektrolyte. Bei ausschließlicher Verminderung $\operatorname{der}$ Zahl freier lonen würde nicht nur $\wedge$, sondern auch $\Delta$ (der osmotische Druck) abnehmen.

5. $\wedge$ und $\triangle$ fallen leicht, s bleibt ganz oder nahezu konstant: Die Konzentration der freien (osmotisch aktiven) lonen hat infolge gesteigerter Adsorption von seiten der Kolloide abgenommen.

6. Geichzeitiges Absinken von $\wedge, \Delta$ und $\mathrm{s}$ ist eine Folge von Elektrolytverlust.

7. $\wedge$ und $\Delta$ nehmen stark $a b, s$ steigt : Der Kolloidgehalt hat bedeutend zugenommen.

8. A nimmt stark zu, s stark ab, $\triangle$ bleibt konstant: Leichter Schwund von dissoziationshemmenden Nichtelektrolyten, starke Abnahme des Kolloidgehaltes.

9. $\Lambda$ steigt leicht an, $\Delta$ und s fallen beträchtlich: Bedeutender Verlust an Nichtelektrolyten, wodurch die Dissoziation gehoben wird (eventuell auch geringfiigiges Verschwinden von Kolloiden).

10. Gleichzeitige Zunahme von $\Lambda, \Delta$ und s bedeutet Erhöhung der lonenkonzentration.

11. $\Lambda$ und $s$ andern sich nicht, $\eta$ nimmt zu: Zunahme der Zahl von viskositătserhöhenden lonen bei gleichzeitigem Verschwinden von die innere Reibung der Kolloideher absetzenden lonen.

12. $\Lambda$ bleibt gleich, $\eta$ und s nehmen zu: Kann nur Ansteigen des Kolloid- und Elektrolytgehaltes bedeuten, denn bei alleiniger Zunahme der Kolloide würde $\Lambda$ vermindert werden.

13. Wahrend $\wedge$ sich nicht ändert, nimmt $\eta$ ab und $s$ zu: Eine Zunahme kristalloider Serumbestandteile - vielleicht begleitet von Kolloidschwund - hat stattgefunden, auch von Nichtelektrolyten, welche durch Zurückdrăngen der Dissoziation die Anzahl freier Ionen auf ursprünglicher Hohe halten.

14. $\wedge$ fallt, $\eta$ und $s$ andern nicht: $A b-$ nahme der Elektrolytkonzentration, verbunden mit Zunahme der Konzentration der Nichtelektrolyte, kann die Ursache sein. Verlust von viskositätserhöhenden lonen, vergesellschaftet mit leichtem Anwachsen der Menge der Kolloide, kann einen ahnlichen Effekt haben. Schlieflich kann blowe vermehrte Ionenadsorption von seiten der Serumkolloide imstande sein, ohne Viskositätsänderung die Leitfahigkeit zu vermindern.

15. Abnahme von $A$ und $s$ bei gleichbleibendem $\eta$ bedeutet Verminderung der Zahl freier Ionen, und zwar hauptsächlich solcher, die für die Viskosität nicht oder von wenig Belang sind. Abnahme des Gehaltes an viskositatserhöhenden und herabsetzenden Ionen würde denselben Effekt haben.

16. Abnahme von $\wedge$ und $\eta$ bei gleichbleibendem $s$ kann eine Folge von Ionenadsorption oder von lonenschwund aus detn Serum, verbunden mit Anwachsen der Nichtelektrolytkonzentration sein.

17. $\wedge, \eta$ und $s$ fallen, wenn beträchtlicher Elektrolytverlust stattfindet (eventuell auch bei Kolloidverlust, der jedoch von der Abnahme der Elektrolytkonzentration in seinem Einflub auf die Leitfăhigkeit äberholt werden würde).

18. Abnahme von $\Lambda$ und $\Delta$ bei gleichzeitigem Anwachsen von $s$ und $\eta$ kann kaum anders als durch eine Bereicherung des Serums an kolloiden Komponenten erklart werden.

19. Verminderung von $\wedge$ und $s$ bei gleichzeitigem Ansteigen von $\eta$ bedeutet Konzentrationsabnahme eines spezifisch die Leitfăhigkeit herabsetzenden lons.

20. Ansteigen von $A$, während $\eta$ und s unverăndert bleiben, kann vielleicht auch durch Freiwerden von Ionen infolge Dissoziation der Ionenproteidverbindungen erklärt werden.

21. $\wedge$ und $\eta$ nehmen zu, s bleibt konstant : Zurücktreten von viskositätserniedrigenden Nichtelektrolyten unter Anwachsen des Elektrolytgehaltes, oder Schwund von Nichtelektrolyten bei gleichzeitigem Auftreten von viskositatserhöhenden lonen können die Ursache dieser Erscheinung sein.

22. Erhöhung von $\wedge$ bei Erniedrigung von $\eta$ und unverändertem $s$ bedeutet Zunahme von die Viskosităt herabsetzenden lonen, begleitet rom Verschwinden von anderen lonen oder Nichtelektrolyten, oder aber Zurückgehen des Kolloidund Ansteigen des Elektrolytgehaltes.

23. Anwachsen von $\Lambda$ und $\eta$ unter $A b$ sinken von s bedeutet Verringerung der Menge von viskositătserniedrigenden Nichtelektrolyten, eventuell unter geringer Vermehrung von lonen, welche die innere Reibung erhőhen. 
24. Zunahme von $\wedge$, vereinigt mit Abnahme von $\eta$ und $s$, kann nur durch Nichtelektrolyt- oder Kolloidschwund erklärt werden.

25. Ein allgemeines Ansteigen von $\Lambda, \eta$ und $s$ hat eine Zunahme der Konzentration von lonen, speziell solcher, welche die Viskosităt erhöhen, zur Ursache.

Die Zahl der Kombinationsmöglichkeiten ist natarlich noch nicht erschöpft. Ich habe nur die tatsăchlich beobachteten Falle hier angeführt; diese zeigen jedoch zur Genüge, dab es möglich ist, aus den Resultaten verschiedener, im Verein mit der Leitfahigkeitsmessung gleichzeitig bei ein und demselben Serum angewandter physikalischchemischer Methoden weitgehende Schlüsse zu ziehen auf seine Beschaffenheit und eventuelle pathologisch-physikalische Verănderungen, speziell aber auf das Verhältnis der Elektrolyte zu den kolloiden Komponenten.

\section{Z u sam m e n a ssung.}

In vorliegender Arheit wird gezeigt, daß die vier Kolloidarten, Serumalbumin, Serumglobulin, Gelatine und Saponin, die Leitfahigkeit einer $1 / 2 \mathrm{n}$ Lossung von $\mathrm{NaOH}$ in verschiedenem Grade herabsetzen, und das diese Herabsetzung mit zunehmender Kolloidkonzentration bei Albumin, Globulin und Saponin so abnimmt, dab die Leitfahigkeitskurve leicht konkav ist. Die Ursache dieser Erscheinung, daB (jetzt rockwarts gehend) die Leitfahigkeit bei Verdünnung des Kolloids rascher anwächst als seiner jeweiligen Konzentration entspricht, wird zur Hauptsache in der relativen Erhöhung der lonenkonzentration in der Zwischenflüssigkeit, dann auch in der Vergroßerung der Eigenleitfähigkeit der Teilchen und der verminderten inneren Reibung des Systems gesehen.

An Hand der Experimente werden die im Innern eines Kolloids stattfindenden Vorgänge einer Analyse unterworfen, wobei es sich herausstellt, dab Quellungs- und Adsorptionsprozesse, Oberflächenspannung der Teilchen, Konzentration derselben und der lonen und die Art der letzteren von hervorragender Wichtigkeit sind und dak die Leitfähigkeit des ganzen Systems von diesen Faktoren abhăngig ist. Es wird hervorgehoben, daB solche Prozesse als Folge des Stoffwechsels auch in der Blutflüssigkeit sich abspielen.

Die Leitfahigkeit eines Kolloid-Ionen-Systems wird zur Hauptsache als eine Funktion der lonenzahl und der Kolloidkonzentration betrachtet, wobei aber ausdrücklich bemerkt wird, dab oft, eben infolge der erwăhnten Adsorptionsprozesse und des Mithineinsplelens von Nonelektrolyten, die Leitfăhigkeit zu Fehlschlüssen bezügl. des Verhaltens der Elektrolyte führen kann. Dies zu vermeiden, wird die gleichzeitige Anwendung mehrerer physikalisch-chemischer Methoden im Verein mit der Leitfähigkeitsmessung empfohlen, deren Ergebnisse Rückschlüsse auf die Beschaffenheit bezw. $\mathrm{Zu}$ sammensetzung physiologischer und pathologischer Sera erlauben. Als Beispiele werden dann die Ergebnisse von 25 Fallen gleichzeitiger Anwendung von drei Methoden bei Seren qualitativ mitgeteilt und $z u$ deuten versucht.

\section{Beiträge zur Kenntnis der Emulsionen.}

Von Wa. Ostwald. (Vorläufige Mitteilung.)

Gelegentlich praktischer Arbeiten über Holzimprägnierung stieb ich auf grundsätzliche Fragen über Emulsionen, die ich nach älteren Handversuchen in den letzten Monaten des Jabres $1908 \mathrm{Im}$ Laboratorium für angewandte Chemic zu Leipzig experimentell naher untersuchte. Obwohl es mir nicht moglich ist, schon jetzt die gesamten Ergebnisse meiner Arbeit zu veröffentlichen, morchte ich doch die als zweckmaBig ausprobierte Męthodik und einige grundlegende Tatsachen schildern.

Das Steigen der Teerolpreise machte es nötig, beim Imprăgnieren von Eisenbahnschwellen und Telegraphenstangen mit dem Imprăgniermittel zu sparen, wăhrend man früher im Interesse der Wirksamkeit und Vollständigkeit der Imprägnierung bemüht gewesen war, pro Kubikmeter Holz möglichst viel Oel einzupressen. Diese Teerölersparnis hat kein Bedenken, sobald es gelingt, die Zellwände des Holzes mit Teerol zu imprägnieren, wenn auch dabei die Hohlrătume der Zellen selbst leer bleiben.

Um dies zu erreichen, hat man auber anderen Mitteln versucht, mit wässerigen Teerölemulsionen zu imprägnieren, wobei man zur Förderung der Emulgierung das Oel entweder durch Schwefelsäure ansulfonierte, oder Ammoniakharzseifen, harzesterschwefelsaures Alkali, Teerőlseifen usw. benutzte (vgl. z. B. D. R. P. 151020,117565 , 117263 ). 